\title{
Mollification formulas and implicit smoothing
}

\author{
R. K. Beatson \\ H.-Q. Bui
}

Research Report UCDMS 2003/19, December 2003

Department of Mathematics and Statistics, University of Canterbury, Christchurch, NEW ZEALAND

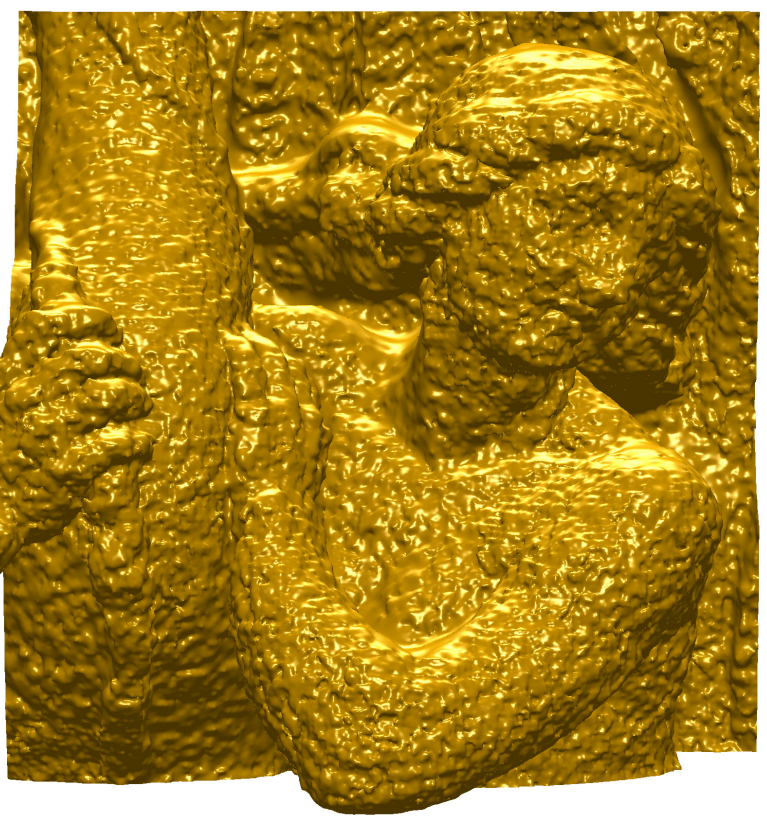

Original unsmoothed fit

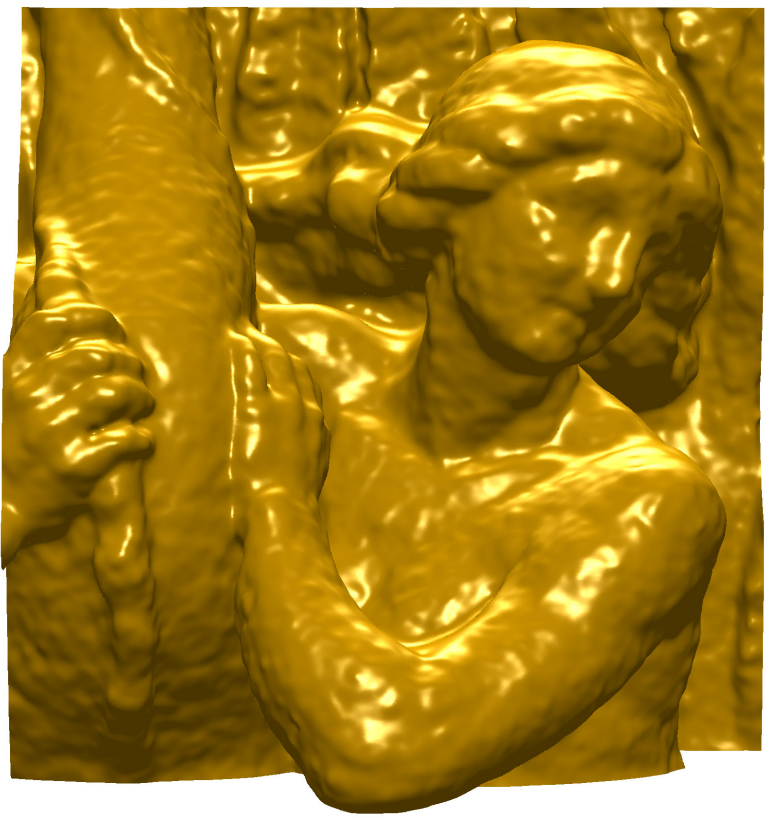

Smoothed with $c=10 \mathrm{~mm}$

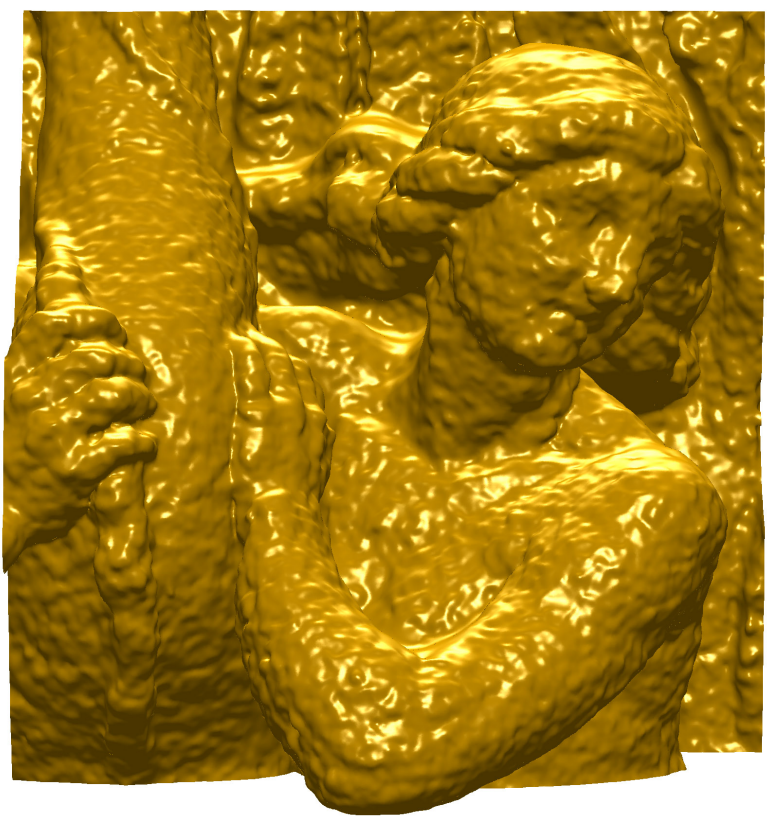

Smoothed with $c=5 \mathrm{~mm}$

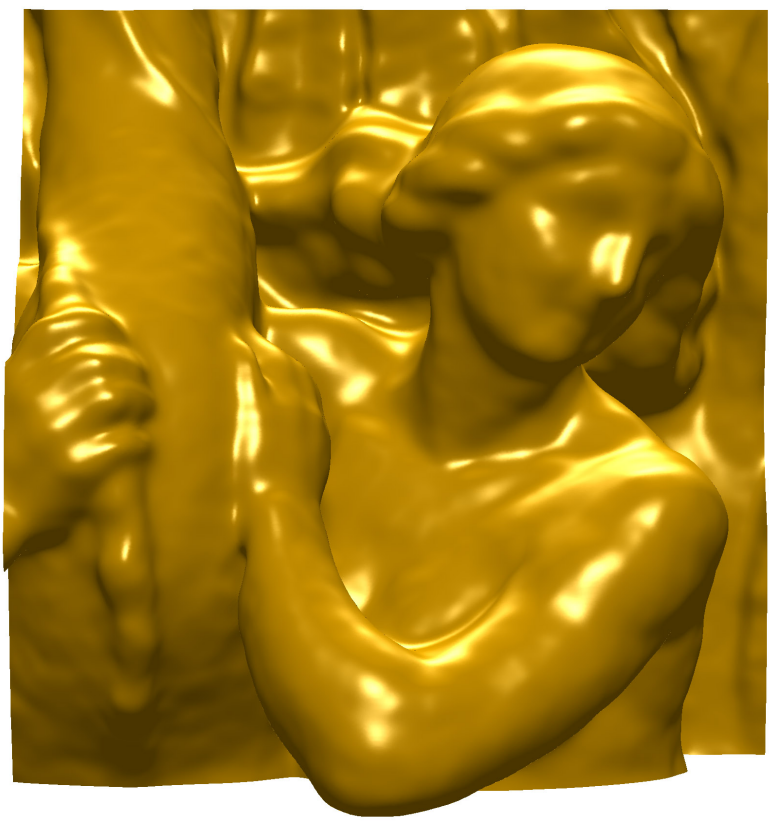

Smoothed with $c=20 \mathrm{~mm}$

Figure 1: Implicit smoothing applied to a noisy Lidar scan. 


\section{Introduction}

This paper develops some mollification formulas involving convolutions between popular radial basis function (RBF) basic functions $\Phi$, and suitable mollifiers $k$. Polyharmonic spline, scaled Bessel kernel (Matern function) and compactly supported basic functions are considered. An application which motivated the development of the formulas is a technique called implicit smoothing. This computationally efficient technique smooths a previously obtained RBF fit by replacing the basic function $\Phi$ with a smoother version $\Psi$ during evaluation. In the case of the polyharmonic spline basic functions the smoothed basic function is a generalised multiquadric or shifted thin-plate spline (at least up to a polynomial).

Special cases of one of the mollification formulas developed here were given in the 1D setting in [Beatson and Dyn 1996]. That paper concerned error estimates for quasi interpolation with $1 \mathrm{D}$ generalised multiquadrics, and showed by elementary methods

$$
\left(\bullet^{2}+c^{2}\right)^{(2 j-1) / 2}=|\bullet|^{(2 j-1) / 2} \star j \frac{(2 j-1) ! !}{(2 j) ! !} c^{2 j}\left(\bullet^{2}+c^{2}\right)^{-(2 j+1) / 2},
$$

for $j \in \mathbb{N}$. A multivariate analog relating $\Phi(x)=|x|$ and the multiquadric $\Psi(x)=\sqrt{x^{2}+c^{2}}$ in $\mathcal{R}^{3}$ has been used to smooth implicit surface fits to lidar and laser scanner data (see Figures 1 and 2, and Section 2). This application is detailed in [Carr et al. 2003]. That paper presents the application but not the mathematics underlying it. Implicit smoothing of globally supported RBFs should also have many other applications. [Fasshauer 1999] has used related basic function substitution techniques as part of a process for the numerical solution of PDEs with compactly supported RBFs.

The purpose of the current paper is to present a mathematical treatment of general versions of these, and related, mollification formulas. Initially our development for the polyharmonic spline case was based on viewing odd powers of $|x|$ as multiples of fundamental solutions of iterated versions of Laplace's equation. As such our treatment was restricted to polyharmonic splines in odd dimensions. Changing to arguments based more directly on generalised functions has enabled many restrictions to be dropped. For example in the case of the results for $|x|^{\beta}$ (Theorem 4.1) there is no longer any restriction on the parity of the dimension, nor any requirement that the power $\beta$ of $|x|$ be odd or integer. Furthermore we develop analogous formulas for polyharmonic splines in even dimension. Related results for scaled Bessel kernels (Matern functions) and compactly supported radial basis functions are also discussed.

Notation: In this paper the Fourier transform is defined as follows

$$
\widehat{f}(\xi):=\int_{\mathcal{R}^{d}} e^{-i x \xi} f(x) d x, \quad f \in L^{1}\left(\mathcal{R}^{d}\right) .
$$

Also, except where explicitly noted, the generalized Fourier transforms that appear are the generalised transforms of the functions viewed as distributions acting on $\mathcal{D}\left(\mathcal{R}^{d} \backslash\{0\}\right)$ rather than on $\mathcal{D}\left(\mathcal{R}^{d}\right)$. This convention simplifies the discussion.

\section{An application - implicit smoothing}

This section concerns an application of the mollification formulas to come to the smoothing of RBF fits. This particular application motivated the development of the formulas. The process will be called implicit smoothing and can be viewed as smoothing an interpolant to noisy data rather than smoothing the data itself.

The process starts with a noisy data set to be approximated. Figure 2 shows one example of such a noisy data set, a "noisy" Lidar

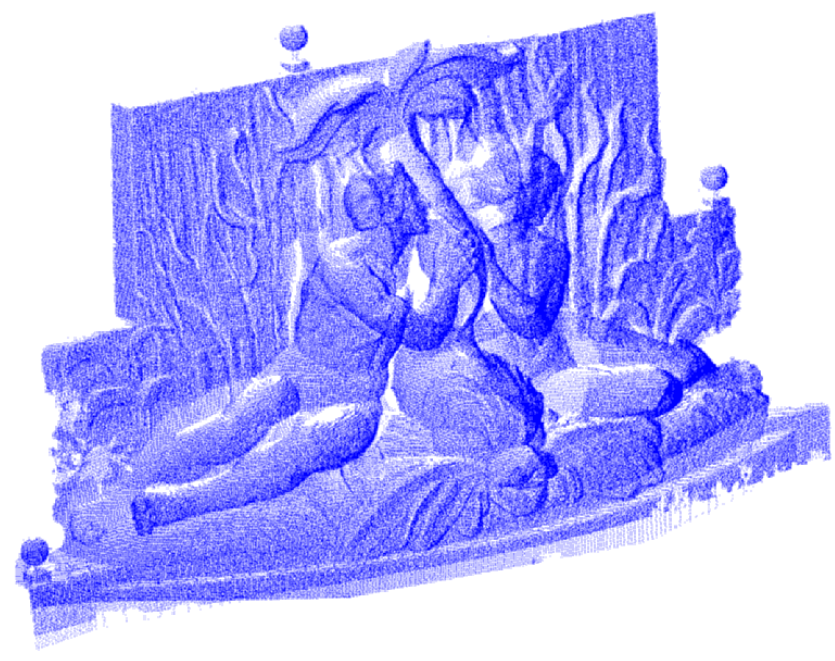

Figure 2: A noisy lidar scan of a statue in Santa Barbara.

scan. Firstly an RBF is fitted to the noisy data yielding an RBF approximation

$$
s(x)=p(x)+\sum_{i=1}^{N} \lambda_{i} \Phi\left(x-x_{i}\right) .
$$

Then the initial RBF approximation is smoothed by convolution with the mollifier $k$ yielding a smoother fit

$$
\widetilde{s}(x)=q(x)+\sum_{i=1}^{N} \lambda_{i} \Psi\left(x-x_{i}\right),
$$

where $q=p \star k$ and $\Psi=\Phi \star k$. Figure 1 shows zoomed in views of the isosurfaces arising when this strategy applied to the noisy Lidar scan of Figure 2. Here $\Phi(x)=|x|$ is the biharmonic spline basic function in $\mathcal{R}^{3}$ and $\Psi(x)=\sqrt{x^{2}+c^{2}}$ is the ordinary multiquadric. In Figure 1 one can clearly see the amount of smoothing increase with the parameter $c$. Figure 3 shows a thin-plate spline fit to data from the Mexican hat function $f(x)=\left(1-x^{2}\right) \exp \left(-x^{2} / 2\right)$ at 400 scattered points in $\mathcal{R}^{2}$. Uniform random noise of magnitude 0.7 has been added to the original Mexican hat height data. Here $\Phi(x)=x^{2} \log |x|$ is the thin-plate spline and $\Psi(x)=\left(x^{2}+c^{2}\right) \log \sqrt{x^{2}+c^{2}}$ is the shifted thin-plate spline. Implicit smoothing has been employed to obtain an approximation to the noise free signal.

For important choices of $\Phi$, and suitable choices of $k$, the smoothed basic function $\Psi$ turns out to be a simple and easy to evaluate, function. Therefore in applying the technique there is no need for any explicit, and computationally expensive, evaluation of convolutions. Rather one fits the initial radial basis function $s$, and then smooths it when evaluating by substituting the smoothed basic function $\Psi$ for the original basic function $\Phi$. Thus the technique can be viewed as smoothing by basic function substitution. In some important cases fast evaluators are available for the smoothed RBF $\widetilde{s}$.

Advantages of the technique are

- No explicit convolution to do.

- There is no requirement that the data or evaluation points be gridded.

- Well understood linear filtering. Fourier transform and absolute moments of the smoothing kernel are known. 


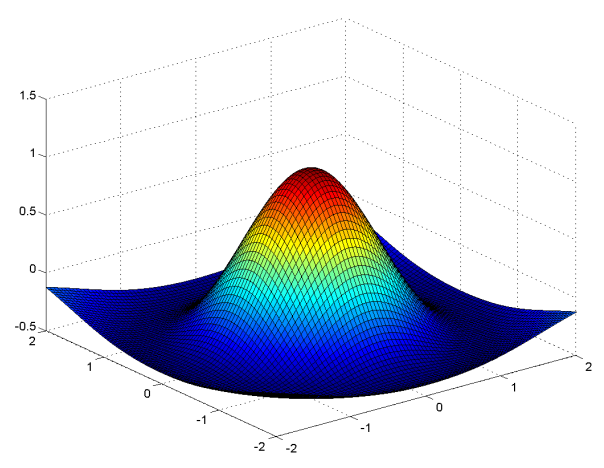

(a) The Mexican hat function

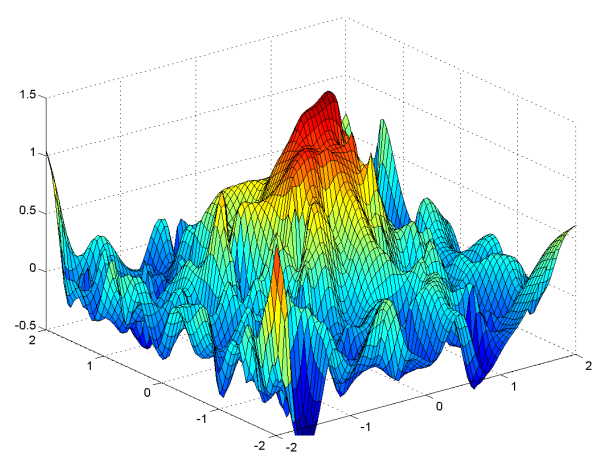

(b) Exact fit to Mexican hat plus noise data

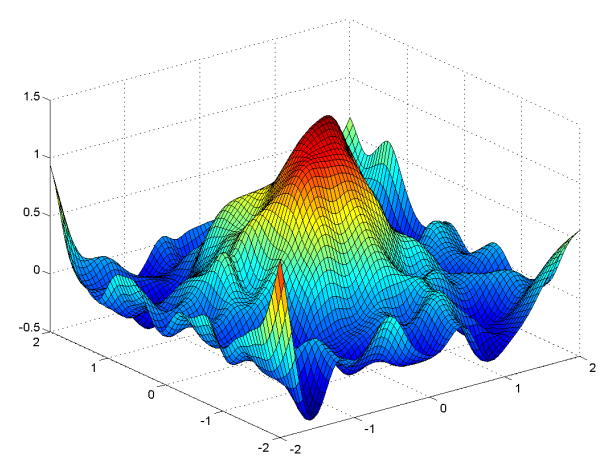

(c) Smoothed with $c=0.2$

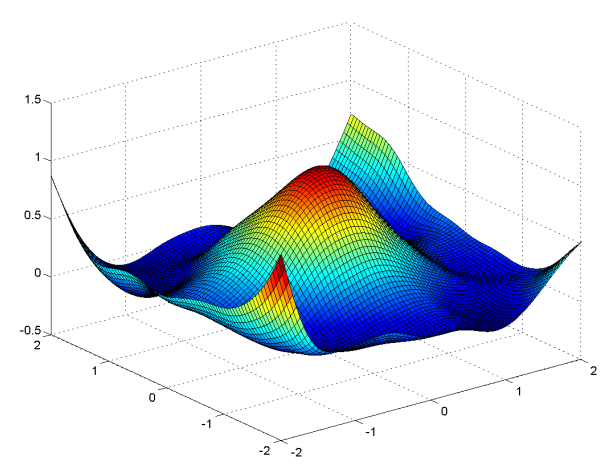

(d) Smoothed with $c=0.6$

Figure 3: Various fits to noisy data created from the Mexican hat function.
- Smoothing can be chosen for appropriate frequency or length scale.

- A posteriori parameter for user to play with - "noise level/frequency" need not be known a priori.

Disadvantages of the method

- A posteriori parameter for user to play with.

- Just linear filtering so will blur sharp features.

\section{Technical lemmas}

The following technical lemmas which deal with distributions and special functions will be needed in later sections.

Lemma 3.1. Identification of a convolution

(i) Let $\beta, \epsilon>0$. Suppose $g \in C\left(\mathcal{R}^{d}\right), g(x)=\mathcal{O}\left(|x|^{\beta}\right)$ as $|x| \rightarrow$ $\infty$, and $k \in L^{\infty}\left(\mathcal{R}^{d}\right), k(x)=\mathcal{O}\left(|x|^{-(d+\beta+\epsilon)}\right)$ as $|x| \rightarrow \infty$. Then $g \star k$ is a $C\left(\mathcal{R}^{d}\right)$ function with $(g \star k)(x)=\mathcal{O}\left(|x|^{\beta}\right)$ as $|x| \rightarrow \infty$.

(ii) Let $\beta, \epsilon, g$ and $k$ be as in part (i). Let $h \in C\left(\mathcal{R}^{d}\right)$ be such that $h(x)=\mathcal{O}\left(|x|^{\beta}\right)$ as $|x| \rightarrow \infty$. Viewing $\widehat{g}$ and $\widehat{h}$ as tempered distributions suppose that there exist functions $G$ and $H$ in $L_{\text {loc }}^{1}\left(\mathcal{R}^{d} \backslash\{0\}\right)$ such that for all test functions $\phi \in$ $\mathcal{D}\left(\mathcal{R}^{d} \backslash\{0\}\right)$

$$
\langle\widehat{g}, \phi\rangle=\int_{\mathcal{R}^{d}} G(\xi) \phi(\xi) d \xi
$$

and

$$
\langle\widehat{h}, \phi\rangle=\int_{\mathcal{R}^{d}} H(\xi) \phi(\xi) d \xi
$$

Further writing $\widehat{k}$ for the classical Fourier transform of $k$ suppose

$$
G(\xi) \widehat{k}(\xi)=H(\xi), \quad \text { for almost all } \xi \neq 0 .
$$

Then

$$
(g \star k)(x)=p(x)+h(x) \quad \text { for all } x \in \mathcal{R}^{d}
$$

where $p$ is a polynomial of degree not exceeding the integer part of $\beta$.

Proof of part (i)

$$
\begin{aligned}
(g \star k)(x) & =\int g(x-y) k(y) d y \\
& =\mathcal{O}\left(\int(1+|x|+|y|)^{\beta}(1+|y|)^{-(d+\beta+\epsilon)} d y\right) \\
& =\mathcal{O}\left((1+|x|)^{\beta} \int(1+|y|)^{\beta}(1+|y|)^{-(d+\beta+\epsilon)} d y\right) \\
& =\mathcal{O}\left((1+|x|)^{\beta}\right) .
\end{aligned}
$$

Now fix $x \in \mathcal{R}^{d}$ and let $\left\{t_{n}\right\}$ be a sequence tending to zero in $\mathcal{R}^{d}$ with $\left|t_{n}\right| \leq 1$ for all $n$. By an analogous argument to that above there is a constant $C$ so that

$$
\left|g\left(x+t_{n}-y\right) k(y)\right| \leq C(2+|x|)^{\beta}(1+|y|)^{-(d+\epsilon)}
$$


for almost all $y$. Hence, applying the Lebesgue dominated convergence theorem,

$$
\lim _{n \rightarrow \infty}(g \star k)\left(x+t_{n}\right)=(g \star k)(x) .
$$

It follows that $g \star k$ is continuous.

\section{Proof of part (ii)}

Below we will view $g \star k$ as a tempered distribution. Let $\phi \in$ $\mathcal{D}\left(\mathcal{R}^{d} \backslash\{0\}\right)$. Hence $\widehat{\phi} \in \mathcal{S}$ the space of rapidly decreasing functions. The growth conditions on $g, k$, and $\widehat{\phi}$ combine to imply that all the iterated integrals below are absolutely convergent, so that the applications of Fubini's Theorem that occur are justified.

$$
\begin{aligned}
& \langle(g \star k), \phi\rangle=\langle g \star k, \widehat{\phi}\rangle \\
& =\iint g(x-y) k(y) d y \widehat{\phi}(x) d x \\
& =\iint g(z) \widehat{\phi}(y+z) d z k(y) d y \\
& =\int\left(\int g(z)\left[e^{-\mathbf{i} y \xi} \phi(\xi)\right] \curlyvee(z) d z\right) k(y) d y \\
& =\int\left\langle g,\left[e^{-\mathbf{i} y \bullet} \phi(\bullet)\right]\right\rangle k(y) d y \\
& =\int\left\langle\widehat{g}, e^{-\mathbf{i} y \bullet} \phi(\bullet)\right\rangle k(y) d y \\
& =\int\left(\int G(\xi) e^{-\mathbf{i} y \xi} \phi(\xi) d \xi\right) k(y) d y \\
& =\iint G(\xi) e^{-\mathbf{i} y \xi} \phi(\xi) k(y) d y d \xi \\
& =\int G(\xi) \phi(\xi)\left(\int e^{-\mathbf{i} y \xi} k(y) d y\right) d \xi \\
& =\int G(\xi) \widehat{k}(\xi) \phi(\xi) d \xi \\
& =\int H(\xi) \phi(\xi) d \xi \\
& =\langle\widehat{h}, \phi\rangle \text {. }
\end{aligned}
$$

Hence $\widehat{g \star k}-\widehat{h}$ is a distribution supported at the origin. Therefore $g \star k=p+h$ where $p$ is a polynomial. The growth of $g \star k$ and $h$ implies that $p$ is of degree at most the integer part of $\beta$.

In the following $B$ is the Beta function

$$
B(z, w):=\int_{0}^{\infty} \frac{t^{z}}{(1+t)^{z+w}} \frac{d t}{t}=\frac{\Gamma(z) \Gamma(w)}{\Gamma(z+w)},
$$

and $\psi$ is the Digamma function $\psi(z):=\Gamma^{\prime}(z) / \Gamma(z)$ (see e.g. [Abramowitz and Stegun 1965]). ${ }^{1}$

Lemma 3.2. Let $c, w, v \in \mathcal{R}$ with $c>0$ and $v>w / 2>0$. Then

$$
\int_{0}^{\infty} \frac{r^{w}}{\left(c^{2}+r^{2}\right)^{v}} \frac{d r}{r}=\frac{c^{w-2 v}}{2} B\left(\frac{w}{2}, v-\frac{w}{2}\right) .
$$

Further

$$
I(w, v):=\int_{0}^{\infty} \frac{r^{w} \log r}{\left(c^{2}+r^{2}\right)^{v}} \frac{d r}{r}
$$

${ }^{1}$ Care is needed in interpreting the literature as many authors use $\psi(z)$ to denote the function $\Gamma^{\prime}(z+1) / \Gamma(z+1)$ instead. See for example [Jones 1982, page 114].

$$
\begin{aligned}
= & B\left(\frac{w}{2}, v-\frac{w}{2}\right) \frac{c^{w-2 v}}{2} \times \\
& \left\{\log (c)+\frac{1}{2} \psi\left(\frac{w}{2}\right)-\frac{1}{2} \psi\left(v-\frac{w}{2}\right)\right\} .
\end{aligned}
$$

and

$$
\begin{aligned}
M(w, v):= & \int_{0}^{\infty} \frac{r^{w} \log \left(r^{2}+c^{2}\right)}{\left(c^{2}+r^{2}\right)^{v}} \frac{d r}{r} \\
= & B\left(\frac{w}{2}, v-\frac{w}{2}\right) \frac{c^{w-2 v}}{2} \times \\
& \left\{2 \log (c)+\psi(v)-\psi\left(v-\frac{w}{2}\right)\right\} .
\end{aligned}
$$

Proof of the first identity. The assumptions on $v$ and $w$ clearly imply the integral is convergent. Substituting $c^{2} t=r^{2}$

$$
\begin{aligned}
\int_{0}^{\infty} \frac{r^{w}}{\left(c^{2}+r^{2}\right)^{v}} \frac{d r}{r} & =\frac{1}{2} \int_{0}^{\infty} \frac{\left(c^{2} t\right)^{w / 2}}{\left(c^{2}+c^{2} t\right)^{v}} \frac{d t}{t} \\
& =\frac{c^{w}}{2 c^{2 v}} \int_{0}^{\infty} \frac{t^{w / 2}}{(1+t)^{v}} \frac{d t}{t} \\
& =\frac{c^{w-2 v}}{2} B\left(\frac{w}{2}, v-\frac{w}{2}\right)
\end{aligned}
$$

Proof of the second identity. Since $\int_{0}^{\infty} \frac{r^{w}|\log r|}{\left(c^{2}+r^{2}\right)^{v}} \frac{d r}{r}<\infty$ we use the Lebesgue Dominated Convergence Theorem and differentiate under the integral sign to obtain

$$
I(w, v)=\frac{d}{d w} \int_{0}^{\infty} \frac{r^{w}}{\left(c^{2}+r^{2}\right)^{v}} \frac{d r}{r} .
$$

Employing (3.1) this implies

$$
\begin{aligned}
& I(w, v)= \frac{d}{d w}\left\{\frac{c^{w-2 v}}{2} B\left(\frac{w}{2}, v-\frac{w}{2}\right)\right\} \\
&= \frac{d}{d w}\left\{\frac{c^{w-2 v}}{2} \frac{\Gamma\left(\frac{w}{2}\right) \Gamma\left(v-\frac{w}{2}\right)}{\Gamma(v)}\right\} \\
&= \frac{1}{2 \Gamma(v)}\left\{\log (c) c^{w-2 v} \Gamma\left(\frac{w}{2}\right) \Gamma\left(v-\frac{w}{2}\right)\right. \\
& \quad+\frac{1}{2} \psi\left(\frac{w}{2}\right) \Gamma\left(\frac{w}{2}\right) c^{w-2 v} \Gamma\left(v-\frac{w}{2}\right) \\
&\left.\quad-\frac{1}{2} c^{w-2 v} \Gamma\left(\frac{w}{2}\right) \psi\left(v-\frac{w}{2}\right) \Gamma\left(v-\frac{w}{2}\right)\right\} \\
&= \frac{B\left(\frac{w}{2}, v-\frac{w}{2}\right)}{2} c^{w-2 v} \times \\
& \quad\left\{\log (c)+\frac{1}{2} \psi\left(\frac{w}{2}\right)-\frac{1}{2} \psi\left(v-\frac{w}{2}\right)\right\}
\end{aligned}
$$

Proof of the third identity. Proceeding as in the proof of the second integral identity

$$
M(w, v)=-\frac{d}{d v} \int_{0}^{\infty} \frac{r^{w}}{\left(c^{2}+r^{2}\right)^{v}} \frac{d r}{r} .
$$

Employing (3.1) this implies

$$
\begin{aligned}
M(w, v) & =-\frac{d}{d v}\left\{\frac{c^{w-2 v}}{2} \frac{\Gamma\left(\frac{w}{2}\right) \Gamma\left(v-\frac{w}{2}\right)}{\Gamma(v)}\right\} \\
& =-\frac{\Gamma\left(\frac{w}{2}\right)}{2}\left\{-2 \log (c) c^{w-2 v} \frac{\Gamma\left(v-\frac{w}{2}\right)}{\Gamma(v)}\right.
\end{aligned}
$$




$$
\begin{array}{r}
+c^{w-2 v} \frac{\psi\left(v-\frac{w}{2}\right) \Gamma\left(v-\frac{w}{2}\right)}{\Gamma(v)} \\
\left.-c^{w-2 v} \frac{\Gamma\left(v-\frac{w}{2}\right) \Gamma^{\prime}(v)}{(\Gamma(v))^{2}}\right\} \\
=\frac{B\left(\frac{w}{2}, v-\frac{w}{2}\right)}{2} c^{w-2 v} \times \\
\left\{2 \log (c)+\psi(v)-\psi\left(v-\frac{w}{2}\right)\right\} .
\end{array}
$$

\section{Mollification formulas for powers of the modulus}

In this section mollification formulas will be developed for powers of the modulus. The flavour of the main result, Theorem 4.1, is that the convolution of $|x|^{\beta}$ against an appropriate inverse multiquadric is the generalised multiquadric $\left(|x|^{2}+c^{2}\right)^{\beta / 2}$. Further, a quantitative Korovkin Theorem, Proposition 4.2, estimates the distance between the original unsmoothed RBF $s$ and the corresponding smoothed RBF $\widetilde{s}=s \star k_{d, \beta, c}$.

Define the generalised multiquadric basic function ( the generalised Fourier transform of a Bessel kernel) as

$$
\Psi_{\beta, c}(x)=\left(|x|^{2}+c^{2}\right)^{\beta / 2}, \quad x \in \mathcal{R}^{d} .
$$

where $c>0$. These functions are most often considered in the case that $\beta$ is a positive odd integer. Clearly $\Psi_{\beta, c}$ can be viewed as a smoothed out version of $|x|^{\beta}$. The results of this section show that the "smoothing out" is actually given by a convolution.

More precisely Theorem 4.1 to come shows that in $\mathcal{R}^{d}$ and for all $\beta>-d$

$$
\Psi_{\beta, c}(\boldsymbol{x})=\left(\Phi_{\beta} \star k_{d, \beta, c}\right)(x),
$$

where $\Phi_{\beta}=|x|^{\beta}$ and the convolution kernel $k_{d, \beta, c}(\boldsymbol{x})$ is the generalised multiquadric with negative index $\Psi_{-\beta-2 d, c}(\boldsymbol{x})$, normalised to have integral one. That is

$$
k_{d, \beta, c}(\boldsymbol{x})=a_{d, \beta} c^{d+\beta} \Psi_{-\beta-2 d, c}(\boldsymbol{x}),
$$

for some constant $a_{d, \beta}$. Write

$$
\sigma_{d}:=2 \pi^{d / 2} / \Gamma(d / 2),
$$

for the surface area of the unit sphere in $\mathcal{R}^{d}$. Then

$$
1=\int_{\mathcal{R}^{d}} k_{d, \beta, c}(x) d x=\sigma_{d} a_{d, \beta} c^{d+\beta} \int_{\mathcal{R}} \frac{r^{d}}{\left(r^{2}+c^{2}\right)^{(\beta+2 d) / 2}} \frac{d r}{r},
$$

implying from equation (3.1) that

$$
a_{d, \beta}=\pi^{-d / 2} \frac{\Gamma((\beta+2 d) / 2)}{\Gamma((\beta+d) / 2)} .
$$

Interpretation of convolution against the kernel as a low pass filter will be aided by the expression (4.8) for its Fourier transform

$$
\widehat{k}_{d, \beta, c}(\xi)=\frac{2^{1-(d+\beta) / 2}}{\Gamma((\beta+d) / 2)} K_{\frac{\beta+d}{2}}(c|\xi|)(c|\xi|)^{(\beta+d) / 2}, \quad \beta>0 .
$$

This Fourier transform is a positive function tending to zero exponentially fast with $|\xi|$. Considering the graph of $\widehat{k}_{d, \beta, c}(|\xi|)$ against $|\xi|$ it is clear that the width of the graph at any fixed height is inversely proportional to $c$. This expresses precisely how the graph of $\widehat{k}_{d, \beta, c}(\xi)$ grows more and more peaked as $c$ increases. Thus convolution with $\widehat{k}_{d, \beta, c}$ will attenuate high frequencies more and more as $c$ increases.
Using equation (3.1) again one has

$$
\begin{aligned}
\int_{\mathcal{R}^{d}}|x|^{\beta} k_{d, \beta, c}(\boldsymbol{x}) d x & =\frac{\int_{\mathcal{R}^{d}}|x|^{\beta} k_{d, \beta, c}(\boldsymbol{x}) d x}{\int_{\mathcal{R}^{d}} k_{d, \beta, c}(\boldsymbol{x}) d x} \\
& =\frac{\int_{\mathcal{R}} \frac{r^{d+\beta}}{\left(c^{2}+r^{2}\right)^{(\beta+2 d) / 2}} \frac{d r}{r}}{\int_{\mathcal{R}} \frac{r^{d}}{\left(c^{2}+r^{2}\right)^{(\beta+2 d) / 2}} \frac{d r}{r}} \\
& =c^{\beta} B\left(\frac{d+\beta}{2}, \frac{d}{2}\right) / B\left(\frac{d}{2}, \frac{d+\beta}{2}\right) \\
& =c^{\beta} .
\end{aligned}
$$

We are particularly interested in the polyharmonic splines. In odd dimension odd powers of the modulus are multiples of fundamental solutions of iterated versions of Laplace's equation. Radial basis functions based on sums of shifts of these fundamental solutions supplemented by polynomials have many wonderful properties. Such polyharmonic splines arise naturally as smoothest interpolants (see [Duchon 1977]) and have performed extremely well in many practical applications (see e.g. [Carr et al. 2001]). A particularly important special case is that of the basic function $\Phi(x)=|x|$ in $\mathcal{R}^{3}$. The corresponding RBFs, which take the form of a linear polynomial plus sums of shifts of the modulus, are called biharmonic splines in $\mathcal{R}^{3}$.

We will now show the mollification formula

Theorem 4.1. For all $\beta$ such that $\Re(\beta)>-d$

$$
\left(\Phi_{\beta} \star k_{d, \beta, c}\right)(x)=\Psi_{\beta, c}(x), \quad \text { for all } x \in \mathcal{R}^{d} .
$$

Remark: This result includes the cases where the power $\beta$ and the dimension $d$ are both odd, and $\Phi_{\beta}(x)=|x|^{\beta}$ is a polyharmonic spline basic function. It also includes many other cases. In particular the range of validity of the formula includes those exceptional $\beta$ 's for which $\Phi_{\beta}$ and $\Psi_{\beta, c}$ are polynomial.

Proof. We note the following generalized Fourier transforms for the relevant functions viewed as distributions acting on $\mathcal{D}\left(\mathcal{R}^{d} \backslash\{0\}\right)$.

$$
\left(\mathcal{F} \Psi_{\beta, c}\right)(\xi)=\frac{2 \pi^{d / 2}}{\Gamma(-\beta / 2)} K_{(d+\beta) / 2}(c|\xi|)\left(\frac{|\xi|}{2 c}\right)^{-(d+\beta) / 2}
$$

for $\beta \notin 2 \mathbb{N}_{0}$, where $K_{(d+\beta) / 2}$ is a modified Bessel function (see [Abramowitz and Stegun 1965, page 374] or [Aronszajn and Smith 1961, page 415]). For $\beta<-d$ this is a classical Fourier transform on $\mathcal{R}^{d}$. Also

$$
\left(\mathcal{F}|\bullet|^{\beta}\right)(\xi)=2^{d+\beta} \pi^{d / 2} \frac{\Gamma((d+\beta) / 2)}{\Gamma(-\beta / 2)}|\xi|^{-d-\beta}
$$

for $\beta \notin\left(-d-2 \mathbb{N}_{0}\right) \bigcup\left(2 \mathbb{N}_{0}\right)$ (see [Gelfand and Shilov 1964, page $363])$.

Then using the normalising constant

$$
a_{d, \beta}=\pi^{-d / 2} \frac{\Gamma((\beta+2 d) / 2)}{\Gamma((\beta+d) / 2)}
$$

defined above and that $K_{\nu}(z)=K_{-\nu}(z)$ we find

$$
\begin{aligned}
\widehat{k}_{d, \beta, c}= & a_{d, \beta} c^{d+\beta} \widehat{\Psi}_{-2 d-\beta, c} \\
= & \pi^{-d / 2} c^{d+\beta} \frac{\Gamma((\beta+2 d) / 2)}{\Gamma((\beta+d) / 2)} \frac{2 \pi^{d / 2}}{\Gamma(-(-2 d-\beta) / 2)} \\
& K_{-(d+\beta) / 2}(c|\xi|)\left(\frac{|\xi|}{2 c}\right)^{(d+\beta) / 2}
\end{aligned}
$$




$$
=\frac{2^{1-(d+\beta) / 2}}{\Gamma((\beta+d) / 2)} K_{\frac{\beta+d}{2}}(c|\xi|)(c|\xi|)^{(\beta+d) / 2}
$$

for $\beta>-d$. It is then clear that

$$
\left(\mathcal{F}|\bullet|^{\beta}\right)(\xi) \widehat{k}_{d, \beta, c}(\xi)=\widehat{\Psi}_{\beta, c}(\xi), \quad \xi \in \mathcal{R}^{d} \backslash\{0\} .
$$

for a set of $\beta$ 's including $0<\beta<1$.

Now fix $c>0,0<\beta<1$, and apply Lemma 3.1. The Lemma implies that

$$
|\bullet|^{\beta} \star k_{d, \beta, c}=p_{\beta, c}+\Psi_{\beta, c}
$$

where $p_{\beta, c}$ is a polynomial of degree 0 and the equality holds pointwise. Considering the point $x=0$ we find

$$
\begin{aligned}
\left(|\bullet|^{\beta} \star k_{d, \beta, c}\right)(0) & =\int_{\mathcal{R}^{d}}|t|^{\beta} \star k_{d, \beta, c}(t) d t \\
& =c^{\beta},
\end{aligned}
$$

where we have used (4.6). Then observing that $\Psi_{\beta, c}(0)=c^{\beta}$ we deduce that the polynomial $p_{\beta, c}$ in (4.9) must be identically zero. We have therefore established that for all $c>0$ and $0<\beta<1$

$$
\left(\Phi_{\beta} \star k_{d, \beta, c}\right)(x)=\Psi_{\beta, c}(x), \quad \text { for all } x \in \mathcal{R}^{d} .
$$

Now fix $c>0$ and $x \in \mathcal{R}^{d}$. The right-hand side of (4.7) is an entire function of $\beta$. The left-hand side is continuous on $\Omega_{d}:=\{\beta$ : $\Re(\beta)>-d\}$ by the Lesbesgue dominated convergence theorem. A standard argument using Morera's theorem then shows that the lefthand side is analytic on $\Omega_{d}$. (4.10) shows that (4.7) holds for $0<$ $\beta<1$. Hence, by analytic continuation, it holds for all $\Re(\beta)>$ $-d$.

The remainder of this section will concern the application of the Theorem above to implicit smoothing.

Recall from (4.6) that

$$
\int_{\mathcal{R}^{d}}|x|^{\beta} k_{d, \beta, c}(\boldsymbol{x}) d x=c^{\beta} .
$$

A routine application of Hölder's inequality then shows that

$$
\int_{\mathcal{R}^{d}}|x|^{\alpha} k_{d, \beta, c}(\boldsymbol{x}) d x<c^{\alpha}, \quad \text { for all } 0<\alpha<\beta .
$$

These expressions for the $\alpha$-th absolute moment of $k_{d, \beta, c}$ clearly quantify the manner in which the kernel becomes peaked as $c$ approaches zero. Loosely speaking they shows that the dominant part of convolution against the kernel is averaging on a length scale of approximately $c$, at least for functions of sufficiently slow growth. Thus we can expect convolution against $k_{d, \beta, c}$ to lose, or smooth, detail at this length scale.

A more precise statement about the error between the original RBF $s$ and its smoothed version $\widetilde{s}$ is implied by the quantitative Korovkin theorem we are about to present. See the last paragraph of this section for the details.

Given a uniformly continuous function $g: \mathcal{R}^{d} \rightarrow \mathcal{R}$ define its uniform norm modulus of continuity

$$
\omega\left(g, \mathcal{R}^{d}, \delta\right):=\sup _{x, y \in \mathcal{R}^{d}:|x-y| \leq \delta}|g(x)-g(y)| .
$$

Let $f \in C^{\ell}\left(\mathcal{R}^{d}\right)$ be a function with all $\ell$-th order partials uniformly continuous on $\mathcal{R}^{d}$. Define the $\ell$-th order directional derivative of $f$ at $x$ in the direction of $u, f_{u}^{(\ell)}(x)$, as the $\ell$-th derivative of the univariate function $g(t)=f(x+t u)$, at $t=0$. The joint modulus of continuity of all directional derivatives of order $\ell$ of $f$ is given by

$$
\Omega\left(f^{(\ell)}, \mathcal{R}^{d}, \delta\right):=\sup _{|u|=1} \omega\left(f_{u}^{(\ell)}, \mathcal{R}^{d}, \delta\right) .
$$

For a discussion of the properties of this modulus of continuity see [Beatson and Light 1993]. Given $\ell \in \mathbb{N}_{0}$ and $0<\alpha \leq 1$ we will say the total derivative $f^{(\ell)}$ is in $\operatorname{Lip}_{M}(\alpha)$ if there exists a positive a constant $M$ such that

$$
\Omega\left(f^{(\ell)}, \mathcal{R}^{d}, \delta\right) \leq M \delta^{\alpha}, \quad \text { for all } 0<\delta<\infty .
$$

For each multiindex $\gamma$ adopt the usual notation defining $|\gamma|=\|\gamma\|_{1}$ and the normalized mononomial

$$
V_{\gamma}(x)=\frac{1}{\gamma !} x^{\gamma}=\frac{1}{\gamma_{1} ! \gamma_{2} ! \cdots \gamma_{d} !} x_{1}^{\gamma_{1}} x_{2}^{\gamma_{2}} \cdots x_{d}^{\gamma_{d}}
$$

With this notation in hand we can state the following folklore quantitative Korovkin Theorem. We include the simple proof for the sake of completeness, and also because we do not know of a convenient reference.

Proposition 4.2. Let $\ell \in \mathbb{N}_{0}, 0<\beta \leq 1$, and $B>0$. For each $c>0$ let $k_{c}: \mathcal{R}^{d} \rightarrow \mathcal{R}$ be a bounded function such that

$$
\begin{gathered}
\int_{\mathcal{R}^{d}} t^{\gamma} k_{c}(t) d t=\delta_{0,|\gamma|}, \quad 0 \leq|\gamma| \leq \ell, \\
\int_{\mathcal{R}^{d}}|t|^{\ell+\beta}\left|k_{c}(t)\right| d t \leq B c^{\ell+\beta} .
\end{gathered}
$$

Then for all $f \in C^{\ell}\left(\mathcal{R}^{d}\right)$ with $\ell$-th total derivative in $\operatorname{Lip}_{M}(\beta)$ and all $c>0$

$$
\left\|f \star k_{c}-f\right\|_{\infty} \leq \frac{B M}{\ell !} c^{\ell+\beta} .
$$

Proof. Taylor's theorem with integral remainder for univariate functions implies

$$
\left|g(x-t)-\sum_{j=0}^{\ell} g^{(j)}(x) \frac{(-t)^{j}}{j !}\right| \leq \frac{|t|^{\ell}}{\ell !} \omega\left(g^{(\ell)},|t|\right), \quad x, t \in \mathcal{R} .
$$

Applying this along the line segment joining $x$ and $x-t$ in $\mathcal{R}^{d}$ we find the multivariate Taylor theorem in the form

$$
\left|f(x-t)-\sum_{|\gamma| \leq \ell}\left(D^{\gamma} f\right)(x) V_{\gamma}(-t)\right| \leq \frac{|t|^{\ell}}{\ell !} \Omega\left(f^{(\ell)}, \mathcal{R}^{d},|t|\right) .
$$

Hence using the hypotheses

$$
\begin{aligned}
\mid\left(f \star k_{c}\right)(x) & -f(x)|=| \int_{\mathcal{R}^{d}}(f(x-t)-f(x)) k_{c}(t) d t \mid \\
& =\left|\int_{\mathcal{R}^{d}}\left(f(x-t)-\sum_{|\gamma| \leq \ell}\left(D^{\gamma} f\right)(x) V_{\gamma}(-t)\right) k_{c}(t) d t\right| \\
& \leq \int_{\mathcal{R}^{d}} \frac{|t|^{\ell}}{\ell !} \Omega\left(f^{(\ell)}, \mathcal{R}^{d},|t|\right)\left|k_{c}(t)\right| d t \\
& \leq \frac{M}{\ell !} \int_{\mathcal{R}^{d}}|t|^{\ell+\beta}\left|k_{c}(t)\right| d t \leq \frac{B M}{\ell !} c^{\ell+\beta} .
\end{aligned}
$$

As a first application of Theorem 4.1 and Proposition 4.2 consider implicit smoothing of an RBF of form (2.1) when $\Phi(x)=|x|$ and $p$ is of degree 1. Implicit smoothing of a surface in $\mathcal{R}^{3}$ modelled with such biharmonic spline is illustrated in Figures 1 and 2 . Then the unsmoothed function $s \in \operatorname{Lip}_{M}(1)$ where

$$
M=\sup _{x \in \mathcal{R}^{d} \backslash\left\{x_{i}: 1 \leq i \leq N\right\}}|\nabla s(x)| .
$$


Convolving against $k_{d, 1, c}$ we note that linear polynomials are preserved so that the smoothed RBF (2.2) takes the special form

$$
\widetilde{s}(x)=p(x)+\sum_{i=1}^{N} \lambda_{i} \Psi_{1, c}\left(x-x_{i}\right) .
$$

Applying the Korovkin theorem Proposition 4.2, using that $\int_{\mathcal{R}^{d}}|x| k_{d, 1, c}(x) d x=c$ by (4.6), we see that $\|s-\widetilde{s}\|_{\infty} \leq M c$.

\section{Radial functions}

This section outlines some known fundamental properties of radial functions.

A function $f: \mathcal{R}^{d} \rightarrow \mathcal{R}$ is radial if there is a univarate function $g$ such that $f(x)=g(|x|)$ for all $x$.

Lemma 5.1. Let $f, k: \mathcal{R}^{d} \rightarrow \mathcal{R}$ be such that the integral defining $(f \star k)(x)$ is absolutely convergent for all $x$. If $f$ and $k$ are radial then so is $f \star k$.

Proof. Given $x \in \mathcal{R}^{d}$ choose a rotation matrix $Q$ so that $Q x=$ $|x| e_{1}$ where $e_{1}$ is the vector $(1,0, \ldots, 0)$. Then

$$
\begin{aligned}
(f \star k)(x) & =\int_{\mathcal{R}^{d}} f(x-t) k(t) d t \\
& =\int_{\mathcal{R}^{d}} f(Q x-Q t) k(Q t) d t \\
& =\int_{\mathcal{R}^{d}} f\left(|x| e_{1}-s\right) k(s) d s \\
& =(f \star k)\left(|x| e_{1}\right) .
\end{aligned}
$$

Given a polynomial $p: \mathcal{R}^{d} \rightarrow \mathcal{R}$ write it in terms of the monomial basis as $p(x)=\sum_{\alpha \in \mathbb{N}_{0}^{d}} a_{\alpha} x^{\alpha}$. Define the homogeneous part of degree $j$ of $p, H_{j} p$ by $\left(H_{j} p\right)(x)=\sum_{|\alpha|=j} a_{\alpha} x^{\alpha}$.

Lemma 5.2. Let $p: \mathcal{R}^{d} \rightarrow \mathcal{R}$ be a polynomial which is also radial. Then

(a) All the homogeneous parts $H_{j} p$ of $p, j=0,1, \ldots$, are also radial.

(b) There is a univariate polynomial q such that

$$
p(x)=q(|x|), \quad \text { for all } x .
$$

Proof of $(a)$. Suppose that $p$ is radial yet at least one homogeneous part of $p$ is not. Let $H_{m} p$ be the first non radial homogeneous part of $p$. Then there exist $x, y$ with $|x|=|y|=1$ but $\left(H_{m} p\right)(x) \neq$ $\left(H_{m} p\right)(y)$. Now let

$$
e=p-\sum_{k=0}^{m-1}\left(H_{k} p\right)=\sum_{k=m}^{\infty}\left(H_{k} p\right) .
$$

Then

$$
e(r x)-e(r y)=r^{m}\left(\left(H_{m} p\right)(x)-\left(H_{m} p\right)(y)\right)+\mathcal{O}\left(r^{m+1}\right),
$$

as $r \rightarrow 0^{+}$implying $e(r x) \neq e(r y)$ for all sufficiently small $r>0$. But $e$ is radial by choice of $m$. Contradiction.

Proof of (b) From part (a) if $j$ is odd then $H_{j} p$ is both odd and radial, therefore identically zero. Hence writing $e_{1}$ for the unit vector $(1,0, \ldots, 0)$ and using part (a) again

$$
p(x)=\sum_{j=0}^{\infty}\left(H_{2 j} p\right)(x)
$$

$$
\begin{aligned}
& =\sum_{j=0}^{\infty}\left(H_{2 j} p\right)\left(|x| e_{1}\right) \\
& =\sum_{j=0}^{\infty}|x|^{2 j}\left(H_{2 j} p\right)\left(e_{1}\right) \\
& =\sum_{j=0}^{\infty} b_{2 j} r^{2 j}, \quad r=|x|,
\end{aligned}
$$

where $b_{2 j}=\left(H_{2 j} p\right)\left(e_{1}\right)$.

\section{Mollification formulas for functions of the form $r^{2 j} \log r$}

In this section mollification formulas are developed for the generalised thin-plate spline basic function $|x|^{2 j} \log |x|, j \in \mathbb{N}$. The flavour of the main result Theorem 6.1 is that convolution of the generalised thin-plate basic function against a certain inverse multiquadric yields the corresponding shifted thin-plate spline $\left(|x|^{2}+c^{2}\right)^{j} \log \sqrt{|x|^{2}+c^{2}}$, plus a polynomial of degree $2 j-2$.

In even dimension even powers of the modulus multiplied by $\log |x|$ are fundamental solutions of iterated versions of Laplace's equation. In particular RBFs taking the form of a linear polynomial plus a sum of shifts of $x^{2} \log |x|$ are the biharmonic RBFs in $\mathcal{R}^{2}$. These thin-plate splines can be shown to be the solutions of various smoothest interpolation and penalized smoothing problems (see e.g. [Duchon 1977], [Wahba 1990]) and have proved very successful in many scattered data fitting applications, see for example [Hutchinson and Gessler 1994]. For such functions we will show mollification formulas of the form

$$
|\bullet|^{\beta} \log |\bullet| \star k_{d, \beta, c}=\left(\bullet^{2}+c^{2}\right)^{\beta / 2} \log \sqrt{\bullet^{2}+c^{2}}+p_{\beta},
$$

where $\beta \in 2 \mathbb{N}, k_{d, \beta, c}$ is as in(4.3), and $p_{\beta}$ is a polynomial depending on $d, \beta$ and $c$. The first function on the right above

$$
\Xi_{\beta, c}(x):=\left(|x|^{2}+c^{2}\right)^{\beta / 2} \log \left(|x|^{2}+c^{2}\right)^{1 / 2}, \quad x \in \mathcal{R}^{d} .
$$

is the shifted thin-plate spline basic function of Dyn, Levin and Rippa. Some properties of these functions can be found in [Dyn et al. 1986] and [Dyn 1989].

In contrast to the case of smoothing $|x|^{\beta}$ discussed in Section 4 a nonzero polynomial part does arise in smoothing $|x|^{2 j} \log |x|$. For example the convolution on the left of (6.1) for $d=2,2 j=2$, and $c=1$ evaluated at zero can be rewritten using polar coordinates as

$$
\frac{2}{\pi} \int_{0}^{\infty} 2 \pi r r^{2} \log (r)\left(r^{2}+1\right)^{-3} d r
$$

This equals $1 / 2$, while $\left(r^{2}+1\right) \log \left(r^{2}+1\right)$ evaluated at $r=0$ is 0 . This direct calculation for the special case $d=2$ is in agreement with the general formula (6.5) which we are about to prove.

Explicitly we will show

Theorem 6.1. For $j \in \mathbb{N}$

$$
\left\{\left(|\bullet|^{2 j} \log |\bullet|\right) \star k_{d, 2 j, c}\right\}(x)=\Xi_{2 j, c}(x)+p_{2 j}(x)
$$

where $p_{2 j}$ is a radial polynomial of degree $2 j-2$. Writing $p_{2 j}$ in the form

$$
\begin{array}{r}
p_{2 j}(x)=b_{2 j, 0}+b_{2 j, 2}|x|^{2}+\cdots+b_{2 j, 2 j-2}|x|^{2 j-2}, \\
b_{2 j, 0}=c^{2 j}\left\{\frac{1}{(2 j-2)+d}+\frac{1}{(2 j-4)+d}+\cdots+\frac{1}{d}\right\},
\end{array}
$$


and in particular

$$
b_{2,0}=\frac{c^{2}}{d} .
$$

Proof. Start with the the mollification formula of Theorem 4.1

$$
\left(|x|^{2}+c^{2}\right)^{\beta / 2}=a_{d, \beta} c^{d+\beta} \int_{\mathcal{R}^{d}}|y|^{\beta}\left(|x-y|^{2}+c^{2}\right)^{-(\beta+2 d) / 2} d y
$$

for all $x \in \mathcal{R}^{d}$ and all $\Re(\beta)>-d$. Differentiating both sides with respect to $\beta$ yields

$$
\begin{aligned}
& \left(|x|^{2}+c^{2}\right)^{\beta / 2} \log \left(|x|^{2}+c^{2}\right)^{1 / 2} \\
& =\left(\frac{d}{d \beta} a_{d, \beta} c^{d+\beta}\right) \int_{\mathcal{R}^{d}}|y|^{\beta}\left(|x-y|^{2}+c^{2}\right)^{-(\beta+2 d) / 2} d y \\
& \quad+a_{d, \beta} c^{d+\beta} \int_{\mathcal{R}^{d}}|y|^{\beta} \log |y|\left(|x-y|^{2}+c^{2}\right)^{-(\beta+2 d) / 2} d y \\
& \quad-a_{d, \beta} c^{d+\beta} \int_{\mathcal{R}^{d}}|y|^{\beta}\left(|x-y|^{2}+c^{2}\right)^{-(\beta+2 d) / 2} \times \\
& =A_{\beta}+B_{\beta}-C_{\beta} .
\end{aligned}
$$

Calculation of quantity $A_{\beta}$. From (4.5)

$$
\begin{aligned}
& \left\{\frac{d}{d \beta}\left(a_{d, \beta} c^{d+\beta}\right)\right\}-a_{d, \beta} c^{d+\beta} \log c \\
& =c^{d+\beta} \frac{d}{d \beta}\left\{\pi^{-d / 2} \frac{\Gamma((\beta+2 d) / 2)}{\Gamma((\beta+d) / 2)}\right\} \\
& =c^{d+\beta} \frac{1}{2 \pi^{d / 2}}\left\{\frac{\Gamma^{\prime}\left(\frac{\beta+2 d}{2}\right)}{\Gamma\left(\frac{\beta+d}{2}\right)}-\frac{\Gamma\left(\frac{\beta+2 d}{2}\right) \Gamma^{\prime}\left(\frac{\beta+d}{2}\right)}{\Gamma\left(\frac{\beta+d}{2}\right)^{2}}\right\} \\
& =\frac{1}{2} a_{d, \beta} c^{d+\beta}\left\{\psi\left(\frac{\beta+2 d}{2}\right)-\psi\left(\frac{\beta+d}{2}\right)\right\} .
\end{aligned}
$$

Hence employing the mollification formula (4.7) we find

$$
A_{\beta}=\left(|x|^{2}+c^{2}\right)^{\beta / 2}\left\{\log c+\frac{1}{2} \psi\left(\frac{\beta+2 d}{2}\right)-\frac{1}{2} \psi\left(\frac{\beta+d}{2}\right)\right\} \text {. }
$$

Quantity $B_{2 j}$ is the convolution

$$
\left\{\left(|\bullet|^{2 j} \log |\bullet|\right) \star k_{d, 2 j, c}\right\}(x)
$$

we are interested in.

Calculation of quantity $C_{2 j}$.

$C_{2 j}=\frac{1}{2} a_{d, 2 j} c^{d+2 j} \int|x-y|^{2 j}\left(|y|^{2}+c^{2}\right)^{-(d+j)} \log \left(|y|^{2}+c^{2}\right) d y$

Expanding the term $|x-y|^{2 j}=\left(|x|^{2}-2<x, y>+|y|^{2}\right)^{j}$ that occurs above using the Binomial Theorem it is clear that $|x-y|^{2 j}$ a polynomial of degree $2 j$ in $x$ and $y$. Collecting terms in the expansion by degree in $x$

$$
\begin{aligned}
|x-y|^{2 j}= & \left(|x|^{2}-2<x, y>+|y|^{2}\right)^{j} \\
= & |x|^{2 j}-2 j<x, y>|x|^{2 j-2} \\
& \quad+\left(j|x|^{2 j-2}|y|^{2}+2 j(j-1)<x, y>^{2}|x|^{2 j-4}\right)
\end{aligned}
$$$$
+ \text { terms of lower degree in } x \text {. }
$$

Substituting this expansion into the expression for $C_{2 j}$ reveals that $C_{2 j}$ is a polynomial of degree $2 j$ in $x$. From Lemma 5.1 this convolution of radial functions yields a radial function. Hence from Lemma $5.2 C_{2 j}$ is a polynomial of degree $j$ in $|x|^{2}$.
The term involving $|x|^{2 j}$ in the expression $C_{2 j}$ is

$$
\begin{aligned}
& \frac{1}{2} a_{d, 2 j} c^{d+2 j} \int_{\mathcal{R}^{d}}|x|^{2 j}\left(|y|^{2}+c^{2}\right)^{-(d+j)} \log \left(|y|^{2}+c^{2}\right) d y \\
& =|x|^{2 j} \frac{1}{2} a_{d, 2 j} \sigma_{d} c^{d+2 j} \int_{0}^{\infty} r^{d}\left(r^{2}+c^{2}\right)^{-(d+j)} \log \left(r^{2}+c^{2}\right) \frac{d r}{r} \\
& =|x|^{2 j} \frac{1}{2} a_{d, 2 j} \sigma_{d} c^{d+2 j} M(d, d+j) \\
& =|x|^{2 j}\left\{\log (c)+\frac{1}{2} \psi(d+j)-\frac{1}{2} \psi\left(\frac{2 j+d}{2}\right)\right\}
\end{aligned}
$$

where we have employed (3.3).

Combining the expressions for $A_{2 j}$ and $B_{2 j}$, with the expression above for the coefficient of $|x|^{2 j}$ in $C_{2 j}$ we find that the terms in $|x|^{2 j}$ cancel and therefore the convolution has the form given in equation (6.3) of the Theorem.

We proceed to identify the constant part $b_{2 j, 0}$ of the polynomial $p_{2 j}$. It follows from formula (6.3) that

$$
\left(\left(|\bullet|^{2 j} \log |\bullet|\right) \star k_{d, 2 j, c}\right)(0)=\Xi_{2 j, c}(0)+b_{2 j, 0} .
$$

Using the radial symmetry the left hand side can be rewritten as the univariate integral

$$
\sigma_{d} a_{d, 2 j} c^{d+2 j} \int_{r=0}^{\infty} r^{2 j} \log (r)\left(r^{2}+c^{2}\right)^{-(2 j+2 d) / 2} r^{d-1} d r
$$

Applying the notation and results of Lemma 3.2 this becomes

$$
\begin{aligned}
& \frac{2}{B\left(\frac{d}{2}, \frac{2 j+d}{2}\right)} c^{d+2 j} I(d+2 j, j+d) \\
& \quad=\frac{c^{2 j}}{2}\left\{\log \left(c^{2}\right)+\psi\left(j+\frac{d}{2}\right)-\psi\left(\frac{d}{2}\right)\right\} .
\end{aligned}
$$

Observing that $\Xi_{2 j, c}(0)=c^{2 j} \log (c)$ it follows that

$$
b_{2 j, 0}=\frac{c^{2 j}}{2}\left\{\psi\left(j+\frac{d}{2}\right)-\psi\left(\frac{d}{2}\right)\right\} .
$$

Employing the recurrence [Abramowitz and Stegun 1965, (6.3.5) and (6.3.6)]

$$
\psi(n+z)=\frac{1}{(n-1)+z}+\frac{1}{(n-2)+z}+\cdots+\frac{1}{z}+\psi(z)
$$

the expression for $b_{2 j, 0}$ can be rewritten as

$$
b_{2 j, 0}=c^{2 j}\left\{\frac{1}{(2 j-2)+d}+\frac{1}{(2 j-4)+d}+\cdots+\frac{1}{d}\right\},
$$

establishing formula (6.4). Substituting $j=1$ gives the formula (6.5).

As an application of the mollification formula of Theorem 6.1 consider implicit smoothing of an ordinary thin-plate spline of form

$$
s(x)=p_{1}(x)+\sum_{i=1}^{N} \lambda_{i}\left|x-x_{i}\right|^{2} \log \left|x-x_{i}\right|
$$

in $\mathcal{R}^{2}$. Implicit smoothing of such a fit is illustrated in Figure 3. If the RBF coefficients $\lambda_{i}$ satisfy the usual side conditions

$$
\sum_{i=1}^{N} \lambda_{i}=0 \quad \text { and } \quad \sum_{i=1}^{N} \lambda_{i} x_{i}=0,
$$


then the sum of all the constant parts arising from smoothing, $\sum_{i}\left(\lambda_{i} c^{2} / d\right)$, is zero. Thus the smoothed thin-plate spline is

$$
\widetilde{s}(x)=p_{1}(x)+\sum_{i=1}^{N} \lambda_{i}\left(\left|x-x_{i}\right|^{2}+c^{2}\right) \log \sqrt{\left|x-x_{i}\right|^{2}+c^{2}} .
$$

Further, when (6.7) holds, one can form a far field expansion

$$
A \log (|x|)+P_{1}(x)+\frac{P_{2}(x)}{|x|^{2}}+\frac{P_{3}(x)}{|x|^{4}}+\ldots,
$$

of $s(x)$ where $P_{j}$ denotes a polynomial of degree $j$. This expansion converges to $s(x)$ with a geometric rate for all sufficiently large $|x|$. It follows that the gradient $s^{(1)}(x)=\nabla s(x)$ is bounded and hence $s \in \operatorname{Lip}_{M}(1)$ with

$$
M=\sup _{x \in \mathcal{R}^{d}}|\nabla s(x)|
$$

Hence noting from (4.11) that $\int_{\mathcal{R}^{d}}|x|^{\alpha} k_{d, 2, c}(x) d x<c^{\alpha}$ for all $0<\alpha<2$ and applying the Korovkin theorem Proposition 4.2 we find that in this case

$$
\|s-\widetilde{s}\|_{\infty}<M c .
$$

Consider now using the triharmonic spline in $\mathcal{R}^{2}$ based on sums of shifts of $|x|^{4} \log |x|$ plus quadratics

$$
s(x)=p_{2}(x)+\sum_{i=1}^{N} \lambda_{i}\left|x-x_{i}\right|^{4} \log \left|x-x_{i}\right| .
$$

The triharmonic spline is $C^{3}$. It is natural to use such a triharmonic spline, rather than the usual thin-plate spline, if the function being approximated is smoother, or if a smoother approximation is required.

The usual side conditions for the triharmonic spline are that the coefficients $\lambda_{i}$ are "orthogonal to" quadratics. That is that

$$
\sum_{i=1}^{N} \lambda_{i} q\left(x_{i}\right)=0, \quad \text { for all quadratics } q .
$$

These conditions imply that the polynomial parts arising from smoothing of the weighted shifts of $|\bullet|^{4} \log |\bullet|$ cancel to give the zero polynomial. Therefore the smoothed RBF has the form

$$
\widetilde{s}(x)=q_{2}(x)+\sum_{i=1}^{N} \lambda_{i}\left(\left|x-x_{i}\right|^{2}+c^{2}\right)^{2} \log \sqrt{\left|x-x_{i}\right|^{2}+c^{2}},
$$

where $q_{2}=p_{2} \star k_{d, 4, c}$ will usually differ from $p_{2}$.

Further, when the side conditions (6.8) hold, one can form a far field expansion

$$
Q_{1}(x) \log (|x|)+P_{2}(x)+\frac{P_{3}(x)}{|x|^{2}}+\frac{P_{4}(x)}{|x|^{4}}+\ldots,
$$

of $s(x)$ where $Q_{1}(x)$ is a polynomial of degree 1 , and for all $j, P_{j}$ denotes a polynomial of degree $j$. This expansion converges to $s(x)$ with a geometric rate for all sufficiently large $|x|$. It follows that all the second partials of $s$ are bounded. Hence the first total derivative $s^{(1)} \in \operatorname{Lip}_{M}(1)$ for some constant $M$. Noting from (4.11) that $\int_{\mathcal{R}^{d}}|x|^{\alpha} k_{d, 4, c}(x) d x<c^{\alpha}$ for all $0<\alpha<4$ and applying the Korovkin theorem Proposition 4.2 we find that in this case

$$
\|s-\widetilde{s}\|_{\infty}<M c^{2}
$$

\section{Bessel kernels and Matern functions}

This section discusses mollification formulas for Bessel kernels and the scaled versions known as Matern functions. These mollification formulas can be exploited in an implicit smoothing technique for Matern RBFs.

Recall the Bessel kernels $G_{d, \alpha}$ for $\mathcal{R}^{d}$ given for $\alpha>0$ by

$$
\widehat{G}_{d, \alpha}(\xi)=\left(1+|\xi|^{2}\right)^{-\alpha / 2},
$$

and

$$
G_{d, \alpha}(x)=\frac{1}{\pi^{d / 2} 2^{(d+\alpha-2) / 2} \Gamma(\alpha / 2)} K_{(d-\alpha) / 2}(|x|)|x|^{(\alpha-d) / 2} .
$$

Good references for the many wonderful properties of these functions are [Aronszajn and Smith 1961] and [Donoghue 1969]. The properties that we will need in the following are

- $G_{d, \alpha} \in L^{2}, \alpha>d / 2$.

- $G_{d, \alpha}$ is continuous when $\alpha>d$.

- $G_{d, \alpha}(x)=D_{d, \alpha}|x|^{(\alpha-d-1) / 2} e^{-|x|}(1+o(1))$ as $|x| \rightarrow \infty$. Here $D_{d, \alpha}$ is a constant depending on $d$ and $\alpha$.

- $G_{d, \alpha}$ is positive definite for $\alpha>d$.

- $G_{d, \alpha} \star G_{d, \beta}=G_{d, \alpha+\beta}, \quad \alpha, \beta>0$.

The Bessel kernels are also basic functions corresponding to natural smoothest interpolation problems. [Schaback 1993] and [Schaback 1995] having shown that for $k>d / 2 \mathrm{RBF}$ interpolation with basic function $G_{d, 2 k}$ yields the interpolant minimizing the Sobolev inner product for $W_{2}^{k}\left(\mathcal{R}^{d}\right)$

$$
\|g\|_{W_{2}^{k}\left(\mathcal{R}^{d}\right)}^{2}=\int_{\mathcal{R}^{d}}|\widehat{g}(\omega)|^{2}\left(1+|\omega|^{2}\right)^{k} d \omega
$$

over all sufficiently smooth interpolants.

More explicit forms for some of the Bessel kernels are

$$
G_{d, d+1}(x)=\frac{\pi^{-(d-1) / 2}}{2^{d} \Gamma\left(\frac{d+1}{2}\right)} e^{-|x|},
$$

which is Lipschitz,

$$
G_{d, d+3}(x)=\frac{\pi^{-(d-1) / 2}}{2^{d+1} \Gamma\left(\frac{d+3}{2}\right)}(1+|x|) e^{-|x|},
$$

which is twice continuously differentiable and

$$
G_{d, d+5}(x)=\frac{\pi^{-(d-1) / 2}}{2^{d+2} \Gamma\left(\frac{d+5}{2}\right)}\left(3+3|x|+|x|^{2}\right) e^{-|x|} .
$$

which is four times continuously differentiable. These formulas and others can be derived using [Abramowitz and Stegun 1965, (10.2.15) or (10.2.17)].

Introduce a parameter $c$ by considering the dilated version scaled to have integral 1

$$
M_{d, \alpha, c}(x)=c^{-d} G_{d, \alpha}(x / c) .
$$

We have used the letter $M$ for these functions as in the Statistics literature they are often called Matern functions rather than scaled Bessel kernels (see e.g. [Stein 1999]). From (7.1) and its immediate consequence

$$
\int_{\mathcal{R}^{d}}|x| M_{d, \alpha, c}(x) d x=c \int_{\mathcal{R}^{d}}|x| G_{d, \alpha}(x) d x
$$


it is clear that $c$ is a length scale associated with the kernel $M_{d, \alpha, c}$.

The positive definiteness of the $G_{d, \alpha}$ 's implies that of the $M_{d, \alpha, c}$ 's. Hence the scattered data interpolation problem of finding a Matern RBF of the form

$$
s(\bullet)=\sum_{i} \lambda_{i} M_{d, \alpha, c}\left(\bullet-x_{i}\right)
$$

taking given values $f_{i}$ at a finite number of given distinct points $x_{i}$ is guaranteed to have a unique solution. [Mouat and Beatson 2002] discuss an application of Matern RBFs to the numerical solution of PDEs in which they significantly outperform multiquadrics.

The convolution property of the $G_{d, \alpha}$ 's implies

$$
M_{d, \alpha, c} \star M_{d, \beta, c}=M_{d, \alpha+\beta, c}, \quad \alpha, \beta>0 .
$$

Therefore given an RBF built upon the basic function $M_{d, \alpha, c}$ we can carry out implicit smoothing in the form

$$
\begin{aligned}
s=\sum_{i} \lambda_{i} M_{d, \alpha, c}\left(\bullet-x_{i}\right) & \\
\longrightarrow & \left\{\sum_{i} \lambda_{i} M_{d, \alpha, c}\left(\bullet-x_{i}\right)\right\} \star M_{d, \beta, c} \\
& =\sum_{i} \lambda_{i} M_{d, \alpha+\beta, c}\left(\bullet-x_{i}\right)=: \widetilde{s} .
\end{aligned}
$$

The RBF $s$ based on the kernel $M_{d, \alpha, c}$ is Lipschitz whenever $\alpha \geq$ $d+1$. Hence, the Korovin theorem and the moment estimate (7.2) imply that

$$
\|s-\widetilde{s}\|_{\infty} \leq\left(\int_{\mathcal{R}^{d}}|x| G_{d, \beta}(x) d x\right)\left(\sup _{x \in \mathcal{R}^{d}}|\nabla s(x)|\right) c,
$$

whenever $\alpha \geq d+1$.

\section{Mollification formulas for compactly supported RBFs}

This section discusses mollification formulas for compactly supported RBFs. These mollification formulas can be exploited in implicit smoothing techniques for compactly supported RBFs.

In this section we abuse notation and do not distinguish between radial functions in terms of the dimension of their Euclidean domain. Thus we write $f(x)$ for a radial function which when written as $g(|x|)$ we can consider as having any finite dimensional Euclidean domain $\mathcal{R}^{n}$. Consequently we need to identify the dimensionality of the domain differently and do so by writing $\star_{d}$ for the convolution in $\mathcal{R}^{d}$. Following [Wendland 1995] given an $L_{\infty}$ compactly supported radial function $f$ form from it another radial function $(I f)$ by defining (viewing $f$ as a function of the variable $r=|x|)$

$$
(I f)(t)= \begin{cases}\int_{s=t}^{\infty} s f(s) d s, & 0 \leq t<\infty \\ (I f)(-t), & t \text { otherwise }\end{cases}
$$

Also define for $t>0$

$$
(D f)(t)=-\frac{1}{t} \frac{d}{d t} f(t) .
$$

In the interior of intervals of continuity of $f$ the fundamental theorem of calculus implies

$$
(D I f)(t)=f(t)
$$

Then Wendland derives from the work of [Schaback and Wu 1996] formulas for the convolutions of compactly supported radial functions one of which should read

$$
I\left(f \star_{d+2} g\right)=2 \pi(I f) \star_{d}(I g)
$$

Now let $k_{c}$ be the characteristic function of the ball radius $c$ in $3 \mathrm{D}$, normalised to have integral one. By Bochner's theorem $C_{3}(x)=\left(k_{c} \star_{3} k_{c}\right)(x)$ will necessarily be positive definite. Statisticians call this function the spherical covariance, but it is also known as the Euclid hat. The latter name derives from the analogy with the univariate piecewise linear hat function, or linear B-spline, on a uniform mesh, which is the convolution of two characteristic functions. It follows easily from formula (8.2) that the spherical covariance, normalised to have value 1 at zero, and to be supported on a ball radius 1 , is

$$
C_{3}(x)=\left(1-\frac{3}{2}|x|+\frac{1}{2}|x|^{3}\right)
$$

Other frequently used positive definite compactly supported basic functions in $\mathcal{R}^{3}$ are the Askey function $A$ and the Wendland function $W$ given by

$$
\begin{aligned}
A(r) & =\psi_{2,0}(r)=(1-r)_{+}^{2}, \quad \text { which is Lipschitz } \\
W(r) & =\psi_{3,1}(r)=(1-r)_{+}^{4}(4 r+1), \quad \text { which is } \mathrm{C}^{2} .
\end{aligned}
$$

To use these functions at a length scale $R$ one simply replaces $r$ by $|x| / R$.

We consider implicit smoothing of these basic functions by convolving them with the kernel $k_{c}$ defined above. The resulting function will clearly be supported on $\{x:|x| \leq 1+c\}$. Looking in the Fourier domain we see that the smoothed function will not be positive definite. Positive definiteness is important as it guarantees the existence of solutions to interpolation problems. However, it does not matter for our implicit smoothing application, in which one smooths a previous fitted RBF. If one wants the smoothed basic function to be positive definite then clearly one should choose a compactly supported positive definite function such as the Askey function or spherical covariance, normalised to have integral 1, as the smoothing kernel rather than $k_{c}$.

Maple code based on (8.2) yields piecewise definitions for the smooth approximations $\Psi=\Phi \star_{3} k_{c}$ to these basic functions $\Phi$ when $0<c<1 / 2$. In particular $\left(A \star_{3} k_{c}\right)(r)$ equals

$$
\begin{cases}\frac{1}{10 c^{3}} r^{4}+\left(1-\frac{1}{c}\right) r^{2}+\left(1-\frac{3}{2} c+\frac{3}{5} c^{2}\right), & 0 \leq r<c, \\ r^{2}-2 r+\left(1+\frac{3}{5} c^{2}\right)-\left(\frac{2 c^{2}}{5}\right) \frac{1}{r}, & c \leq r<1-c, \\ \frac{1}{80 c^{3}} r^{5}-\frac{1}{20 c^{3}} r^{4}+\frac{1-3 c^{2}}{16 c^{3}} r^{3}+\frac{1+c}{2 c} r^{2} & \frac{1+6 c^{2}+16 c^{3}+9 c^{4}}{16 c^{3}} r+\frac{1+10 c^{3}+15 c^{4}+6 c^{5}}{20 c^{3}} \\ -\frac{1-5 c^{2}+15 c^{4}++16 c^{5}+5 c^{6}}{80 c^{3}} \frac{1}{r}, \quad 1-c \leq r<1+c .\end{cases}
$$


Also $\left(C_{3} \star_{3} k_{c}\right)(r)$ equals

$$
\left\{\begin{array}{l}
-\frac{1}{140 c^{3}} r^{6}+\frac{3}{40 c}\left(2+\frac{1}{c^{2}}\right) r^{4} \\
+\frac{3 c}{4}\left(1-\frac{1}{c^{2}}\right) r^{2}+\left(1-\frac{9}{8} c+\frac{1}{4} c^{3}\right), 0 \leq r<c \\
\frac{1}{2} r^{3}+\left(\frac{3}{5} c^{2}-\frac{3}{2}\right) r \\
+1+\left(\frac{3}{70} c^{4}-\frac{3}{10} c^{2}\right) \frac{1}{r}, \\
\frac{1}{280 c^{3}} r^{6}-\frac{3\left(1+2 c^{2}\right)}{80 c^{3}} r^{4}+\frac{1+4 c^{3}}{16 c^{3}} r^{3} \\
+\frac{3\left(1-c^{2}\right)}{8 c} r^{2}-\frac{3\left(1+5 c^{2}+10 c^{3}-4 c^{5}\right)}{40 c^{3}} r \\
+\frac{1+8 c^{3}+9 c^{4}-2 c^{6}}{16 c^{3}} \quad c \leq r<1-c, \\
-\frac{3\left(3-14 c^{2}+35 c^{4}+28 c^{5}-4 c^{7}\right)}{560 c^{3}} \frac{1}{r}, \quad 1-c \leq r<1+c .
\end{array}\right.
$$

Finally, $\left(W \star_{3} k_{c}\right)(r)$ equals

$$
\left\{\begin{array}{l}
\frac{-1}{42 c^{3}} r^{8}+\left(\frac{6}{7 c}-\frac{2}{7 c^{3}}\right) r^{6}+\left(-15+9 c+\frac{6}{c}\right) r^{4} \\
+\left(-10+30 c-30 c^{2}+10 c^{3}\right) r^{2} \\
+\left(1-6 c^{2}+10 c^{3}-\frac{45}{7} c^{4}+\frac{3}{2} c^{5}\right), \\
4 r^{5}-15 r^{4}+\left(20+12 c^{2}\right) r^{3} \\
-\left(10+30 c^{2}\right) r^{2}+\left(24 c^{2}+\frac{36}{7} c^{4}\right) r \\
+\left(1-6 c^{2}-\frac{45}{7} c^{4}\right)+\left(\frac{12}{7} c^{4}+\frac{4}{21} c^{6}\right) \frac{1}{r}, \quad c \leq r<1-c, \\
\frac{1}{84 c^{3}} r^{8}-\frac{15}{224 c^{3}} r^{7} \\
+\frac{1-3 c^{2}}{7 c^{3}} r^{6}-\frac{1-15 c^{2}-16 c^{3}}{8 c^{3}} r^{5} \\
-\frac{3\left(2+5 c+3 c^{2}\right)}{2 c} r^{4}+\frac{1+30 c^{2}+160 c^{3}+225 c^{4}+96 c^{5}}{16 c^{3}} r^{3} \\
-5\left(1+3 c+3 c^{2}+c^{3}\right) r^{2} \\
-\frac{3\left(1+7 c^{2}-105 c^{4}-224 c^{5}-175 c^{6}-48 c^{7}\right)}{56 c^{3}} r \\
+\frac{1+14 c^{3}-84 c^{5}-140 c^{6}-90 c^{7}-21 c^{8}}{28 c^{3}} \\
-\frac{5-36 c^{2}+126 c^{4}-420 c^{6}-576 c^{7}-64 c^{9}}{672 c^{3}}, 1-c \leq r<1+c .
\end{array}\right.
$$

These smoothing formulas can be usefully employed when the original unsmoothed compactly supported RBF, $s$, contains many shifts of a single basic function, $\Phi$, with a constant value of the radius $R$. Then the coefficients of the powers of $r=|x| / R$ above can all be precomputed, and the smoothed piecewise basic function, $\Psi$, evaluated reasonably efficiently by Horner's method applied to $r$ or $r^{2}$ as appropriate.

\section{References}

Abramowitz, M., And Stegun, I. A., Eds. 1965. Handbook of Mathematical Functions. Dover, New York.

Aronszajn, N., And Smith, K. T. 1961. Theory of Bessel Potentials. Part 1. Ann. Inst. Fourier., Grenoble 11, 385-475.

BeAtson, R. K., AND Dyn, N. 1996. Multiquadric B-splines. $J$. Approximation Theory 87, 1-24.
Beatson, R. K., And Light, W. A. 1993. Quasi-interpolation by thin plate splines on the square. Constructive Approximation 9, 407-433.

Carr, J. C., Beatson, R. K., Cherrie, J. B., Mitchell, T. J., Fright, W. R., MCCAllum, B. C., And Evans, T. R. 2001. Reconstruction and representation of $3 \mathrm{~d}$ objects with radial basis functions. In Computer Graphics SIGGRAPH 2001 proceedings), 67-76.

Carr, J. C., Beatson, R. K., McCallum, B. C., Fright, W. R., Mclennan, T. J., And Mitchell, T. J. 2003. Smooth surface reconstruction from noisy range data. In GRAPHITE 2003, ACM Press, New York, 119-126.

Donoghue, W. F. 1969. Distributions and Fourier Transforms. Academic Press, New York.

DUCHON, J. 1977. Splines minimizing rotation-invariant seminorms in Sobolev spaces. In Constructive Theory of Functions of Several Variables, Springer-Verlag, Berlin, W. Schempp and K. Zeller, Eds., no. 571 in Lecture Notes in Mathematics, 85100.

DYN, N., LEVIN, D., AND RIPPA, S. 1986. Numerical procedures for surface fitting of scattered data by radial functions. SIAM J. Sci. Stat. Comput. 7, 2, 639-659.

DYN, N. 1989. Interpolation and approximation with radial and related functions. In Approximation Theory VI, Academic Press, C. K. Chui, L. L. Schumaker, and J. D. Ward, Eds., vol. 1, $211-$ 234.

FASSHAUER, G. E. 1999. On smoothing for multilevel approximation with radial basis functions. In Approximation Theory IX, Vanderbilt University Press, C. K. Chui and L. L. Schumaker, Eds., vol. 2, 55-62.

Gelfand, I. M., And ShILOv, G. E. 1964. Generalized Functions, vol. 1. Academic Press, New York.

Hutchinson, M. F., AND Gessler, P. E. 1994. Splines - more than just a smooth interpolator. Geoderma 62, 45-67.

JONES, D. S. 1982. The theory of generalised functions. Cambridge University Press, Cambridge.

Mouat, C. T., And Beatson, R. K. 2002. RBF collocation. Tech. Rep. UCDMS 2002/3, Department of Mathematics and Statistics, University of Canterbury.

SCHABACK, R., AND WU, Z. 1996. Operators on radial functions. J. Computational and Applied Mathematics 71, 257-270.

SCHABACK, R. 1993. Comparison of radial basis function interpolants. In Multivariate approximation from CAGD to wavelets, World Scientific, Singapore, 293-305.

SCHABACK, R. 1995. Multivariate interpolation and approximation by translates of a basis function. In Approximation Theory VIII, Vol. I, World Scientific, Singapore, 491-514.

Stein, M. L. 1999. Interpolation of Spatial Data: Some theory for Kriging. Springer-Verlag, New York.

WahBA, G. 1990. Spline Models for Observational Data. No. 59 in CBMS-NSF Regional Conference Series in Applied Math. SIAM.

WENDLAND, H. 1995. Piecewise polynomial, positive definite and compactly supported radial functions of minimal degree. Advances in Computational Mathematics 4, 389-396. 